\title{
Krank gearbeitet
}

\author{
Menschen, die sich bei der Ausübung ihres Berufs ein Leiden zuziehen, \\ sollten besser geschützt werden, ist das Ziel einer parlamentarischen Initiative. \\ Christian F. Freisleben, Norbert Peter
}

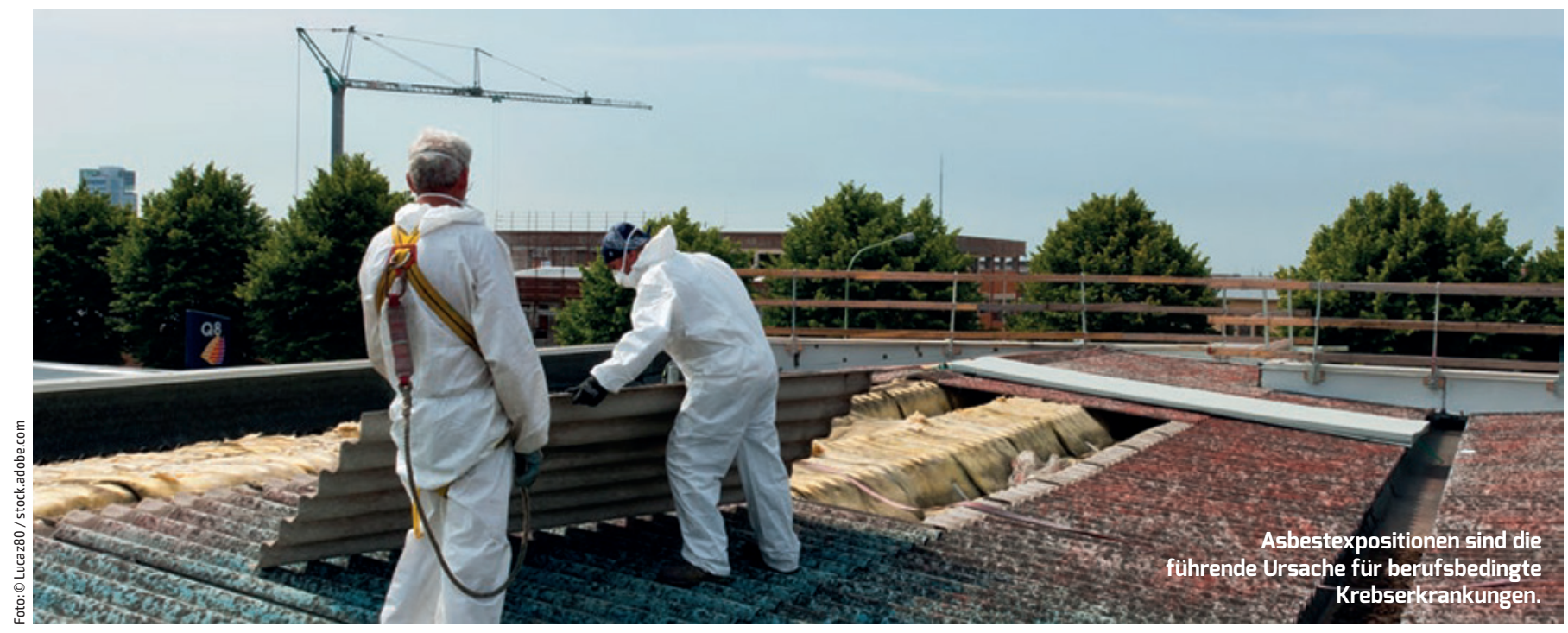

In einem Antrag, der dem Sozialausschuss des Nationalrates zugewiesen wurde, fordern die Abgeordneten Rudolf Silvan und Christian Drobits (SPÖ) eine zeitgemäße Überarbeitung, sprich Erweiterung der Liste der anerkannten Berufskrankheiten. „Das Thema Berufskrankheiten ist in Österreich stark vernachlässigt“, heißt es in dem Antrag. „Im Gegensatz zu Deutschland erfolgt in Österreich keine angemessene Wartung und Anpassung der Berufskrankheitenliste (BK-Liste) an die Veränderung der Arbeitswelt und an neue Erkenntnisse der Wissenschaft." So umfasse die österreichische Berufskrankheitenliste nur 53 Positionen, die deutsche hingegen mehr als 80 .

Ganz ähnlich klingen die Forderungen des Österreichischen Gewerkschaftsbundes (ÖGB), der in einer Presseaussendung darauf hinweist, dass in besagter Liste viele arbeitsbedingte Erkrankungen, wie weißer Hautkrebs, Leiden des Bewegungs- und Stützapparates oder Burn-out, fehlen.

Karl Hochgatterer, Präsident der Österreichischen Akademie für Arbeitsmedizin und Prävention, verweist darauf, dass es in anderen europäischen Ländern noch mehr anerkannte Berufskrankheiten als in Deutschland gibt. „Dahinter stecken nicht nur medizinische Fakten, sondern vielmehr ein sozialpolitischer Diskurs." Eine Voraussetzung für die Definition als Berufskrankheit ist, wie Hochgatterer betont, dass diese Erkrankung in einer Gruppe exponierter Arbeitnehmer doppelt so häufig auftritt wie in der Normalbevölkerung.

„Beim weißen Hautkrebs sehe ich den größten Handlungsbedarf, damit Menschen, die ungeschützt, eventuell über Jahr- zehnte, im Freien arbeiteten, auch zu entsprechenden Leistungen kommen können“, führt Roswitha Hosemann von der Allgemeinen Unfallversicherungsanstalt (AUVA) aus. „In Deutschland ist das seit 2015 eine anerkannte Berufskrankheit, das sollte man übernehmen. Es liegen umfangreiche epidemiologische Erkenntnisse zu den Beziehungen zwischen UV-Strahlung und unterschiedlichen Hautkrebsarten vor."

Menschen, die im Freien arbeiten, sind gegenüber Indoor Workern einer ungleich höheren UV-Belastung ausgesetzt. Dem wird zwar in der Verordnung über persönliche Schutzausrüstung Rechnung getragen, auch durch Zurverfügungstellung von Sonnenschutzcremen für Arbeitnehmer. Um der Entstehung von Plattenepithelkarzinomen und aktinischen Keratosen als Krebsvorstufen vorzubeugen, müssen, wie Hosemann unterstreicht, Arbeitnehmer wie Dachdecker, Maurer und andere, die der Sonne intensiv ausgesetzt sind, aber auch umfassend beraten und geschult werden, damit neben der Verhältnisprävention auch auf das richtige Verhalten hingewiesen wird.

\section{Ohne Meldung kein Gutachten}

Hochgatterer sieht auch noch Ovarialkrebs bei erfolgter Asbestexposition und Wirbelsäulenerkrankungen als wichtige Kandidaten für die Liste der anerkannten Berufskrankheiten in Österreich. Insgesamt ortet er bei berufsbedingtem Krebs viele Hinweise, dass es in Österreich ein großes Problem bei Meldung und Anerkennung gibt: Beispielsweise werden in Deutschland 65 Prozent der Fälle von asbestbedingten Mesotheliomen (Rippen- 
fellkrebs) als beruflich bedingt anerkannt, in Österreich sind es nur 40 Prozent. Dies sei nicht die Schuld der AUVA, diese Tumoren würden nicht gemeldet, und dann könne auch kein Gutachter tätig werden. Hier bräuchte es intensiven Wissens- und Informationsaustausch.

Dass sich bei der Liste der Berufskrankheiten in Österreich in den letzten Jahren wenig bewegt hat, liegt nach Hochgatterers Ansicht auch daran, „dass hierzulande die wissenschaftliche Expertise fehlt. Es gibt nur ein einziges Institut für Arbeitsmedizin an der Universität Wien. In Graz arbeitet der habilitierte Arbeitsmediziner Georg Wultsch mit der dortigen Universität zusammen und setzt entsprechend Lehre um. “ Bis zu einem gewissen Grad ließen sich Daten aus den Zentren für Arbeitsmedizin in Deutschland auf Österreich übertragen, aber es brauche „auch bei uns Wissenschaftler, die dieses Thema für die Politik aufbereiten. Wir sind eher die handelnden Praktiker, die für so etwas wie intensivste Literaturrecherchen kaum Zeit haben", meint Hochgatterer. Dazu ergänzt Hosemann: „In Deutschland gibt es einen ärztlichen Sachverständigenbeirat für Berufskrankheiten. Dieser bewertet und sichtet neue medizinische Erkenntnisse und Forschungsergebnisse, um die Liste von Berufskrankheiten zu aktualisieren und Empfehlungen für neue Berufskrankheiten an das zuständige Ministerium abzugeben. Ein solcher Beirat fehlt in Österreich. Wenn es keine ,Kümmerer' gibt, passiert zu wenig.“

Ist ein Gesundheitsproblem als Berufskrankheit anerkannt, gibt es in Österreich zum einen eine finanzielle Absicherung für Personen, die aus dem Berufsleben ausscheiden müssen. Dazu kommt ein gewisses Angebot im Bereich medizinischer Versor- unter anderem gezielt mit Atemtherapie, körperlichem Aufbautraining und Physiotherapie begegnet. In Großbritannien wird die Aufnahme von Long-COVID in die Berufskrankheitenliste bereits auf politischer Ebene diskutiert.

\section{Hohe Dunkelziffer}

Die Dunkelziffer, vor allem bei beruflich bedingten Krebserkrankungen, sei aufgrund eines Mankos in der Früherkennung groß, sagt Hochgatterer. Auch Formen der nachgehenden Untersuchung, wenn man aus einem exponierten Beruf ausscheidet oder in den Ruhestand tritt, fehlen. Natürlich bräuchte es auch schon bei Menschen, die erwerbstätig sind und krank werden, Arbeitsanamnesen, was sowohl in Betrieben als auch bei Allgemeinme-

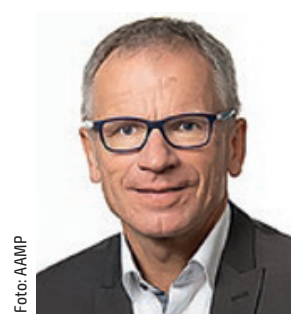

Arbeitsmediziner Karl Hochgatterer: "Es fehlt die wissenschaftliche Expertise."

dizinern noch viel stärker umgesetzt werden müsste. Ursache vieler Krebserkrankungen sind Jahrzehnte zurückliegende Asbestexpositionen: In Österreich werden 120 Krebserkrankungen als beruflich bedingt anerkannt, davon sind 90 bis 100 eine Folge von intensiverem Kontakt mit Asbest in den 1960er-, 70er- und 80er-Jahren bzw. mit damit zusam-

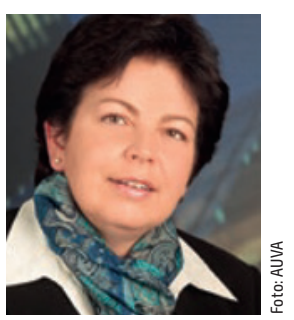
menhängenden Entsorgungsmaßnahmen in den Jahrzehnten darauf. So kann es auch bei aktuellen Restaurierungs- und Umbauprojekten dazu kommen, dass Arbeiter intensiv mit Asbest in Berührung kommen.

Verbesserungsbedarf sieht AUVAÄrztin Hosemann auch beim Wissensstand mancher Kollegen. Sie fordert entsprechende Maßnahmen bereits für die Ausbildung, aber auch für die Praxis: „Beim Verdacht auf eine Berufskrankheit liegt eine Meldepflicht für den Arzt vor. Das Wissen sollte in den Ausbildungen verankert sein, aber auch in den Gesundheitseinrichtungen. Es gibt jetzt ein elektronisches Meldeverfahren über ELGA, das diesen Schritt vereinfacht." Eine Berufsanamnese fehlt oft, was laut Roswitha Hosemann Folgen hat: „Wir wissen alle, dass wir eine hohe Dunkelziffer an Berufskrankheiten haben."

\section{DR. CHRISTIAN F. FREISLEBEN}

freisleben@gesundheitswirtschaft.at

\section{MAG. NORBERT PETER}

norbertpeter@hotmail.com arbeiten, häufig von Handekzemen betroffen.

Aktuell hinterlässt auch das Corona-Virus seine Spuren in der Diskussion. Versicherte, die in Gesundheitsberufen tätig sind und an COVID-19 erkranken, können für drei Wochen in die Klinik Tobelbad zur Rehabilitation aufgenommen werden, wenn sie unter dem Long-COVID-Syndrom leiden. Problemen wie Atemnot unter Belastung, Müdigkeit oder Kopfschmerzen wird dort

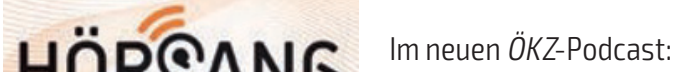 HORCANG Handlungsbedarf bei Berufskrankheiten. von Springer Medizin Wien}

